\title{
An Optimal Guidance Law Applied to Quadrotor Using LQR Method
}

\author{
By Hamidreza JAFARI, Mehran ZAREH, Jafar Roshanian and Amirali NiKKHAH \\ Department of Aerospace Engineering, K. N. Toosi University of Technology, Tehran, Iran
}

(Received February 21st, 2009)

\begin{abstract}
The optimal guidance law of an autonomous four-rotor helicopter, called the Quadrotor, using linear quadratic regulators (LQR) is presented in this paper. The dynamic equations of the Quadrotor are considered nonlinear so to find an LQR controller, it is necessary that these equations be linearized in different operation points. Due to importance of energy consumption in Quadrotors, minimum energy is selected as the optimal criteria.
\end{abstract}

Key Words: Quadrotor, Dynamic Modeling, Guidance, LQR

\author{
Nomenclature \\ $\Omega:$ rotational speed \\ $T$ : torque \\ $\phi$ : roll angle \\ $\theta$ : pitch angle \\ $\psi$ : yaw angle \\ $h$ : altitude \\ $I_{j}$ : inertial moment in $j$ direction
}

\section{Introduction}

The objective of this work is to develop an autonomous guidance and appropriate flight control laws for a Quadrotor, based on a 6 degree-of-freedom (6 DOF) nonlinear dynamic model that make it a fully autonomous aerial vehicle.

Unmanned aerial vehicles (UAV) have attracted significant attention in recent years for both military and civilian applications. Among the different configurations of UAVs, those UAVs with vertical takeoff and landing (VTOL) and hovering (or near hovering) flight capabilities are of particular interest. They are ideal platforms for intelligent surveillance, reconnaissance and rescue. There are many reasons that a Vertical Takeoff and Landing (VTOL) flight platform would be desirable for the exploration of Mars. ${ }^{1)}$ Such as missions the vehicle must automatically be able to take off, cruise, and hover in a specific height. Therefore flight software is necessary. It has the difficult job of directing and controlling the vehicle from its initial position to its final location autonomously. To accomplish this challenging task, the flight software must correctly perform three basic functions: guidance, navigation, and control (GNC). Navigation is the process of determining the vehicle's position, velocity, and attitude in space. Guidance is the process of deciding how to steer to the desired target. Control is the process of implementing the guidance commands to determine appropriate speed of motors.

The Quadrotor concept has been around for a long time. In 1907, the Breguet Brothers built their first helicopter. Their

(C) 2010 The Japan Society for Aeronautical and Space Sciences ungainly quad-rotor Gyroplane No. 1 consisted of four long girders made of steel tubes and arranged in the form of a horizontal cross (Fig. 1). Photographs show the assistance of several men stabilizing and perhaps even lifting the machine. Clearly, the machine never flew completely free because it lacked stability and a proper means of control. ${ }^{2)}$

Recent developments in high density power storage, integrated miniature actuators and MEMS technology sensors have made autonomous miniaturized Quadrotor possible. Nowadays many models of Quadrotor is produced such as Draganflyer X-Pro, ${ }^{3)}$ This vehicle consists of four rotors in total, with two pairs of counter-rotating, fixed-pitch blades located at the four corners of the aircraft, an example of which is shown in Fig. 2.

The interesting feature of the Quadrotor is the use of RPM-based thrust and helicopter control. This has the effect of simplifying the design so that no collective or cyclic controls are necessary. The rotor systems work in pairs, turning opposite directions to cancel each other's torques. In previous designs, as shown in Fig. 3a to begin the helicopter forward motion, the front rotor (1) is slowed down at the same time that the rear rotor (3) is sped up. Yawing is accomplished by slowing one rotor pair while speeding up the other to gain a net torque while still maintaining thrust symmetry about the pitch and roll axes.

The paper is organized as follows. Following this introductory section, Section 2 presents the nonlinear $6 \mathrm{DOF}$ model of the Quadrotor. Section 3 discusses about guidance, navigation, and control (GNC). Section 4 presents the simulation verification of the 6 DOF controller design.

\section{Nonlinear 6 DOF Quadrotor Dynamic Modelling}

The first step before the control development is an adequate dynamic system modeling. Especially for lightweight flying systems, the dynamic model ideally includes the gyroscopic effects resulting from both the rigid body rotation in space, and the four propeller's rotation.

In previous works ${ }^{4,5)}$ two motors are used for pitch and roll commands (Fig. 3a), therefore roll rotation coupled 


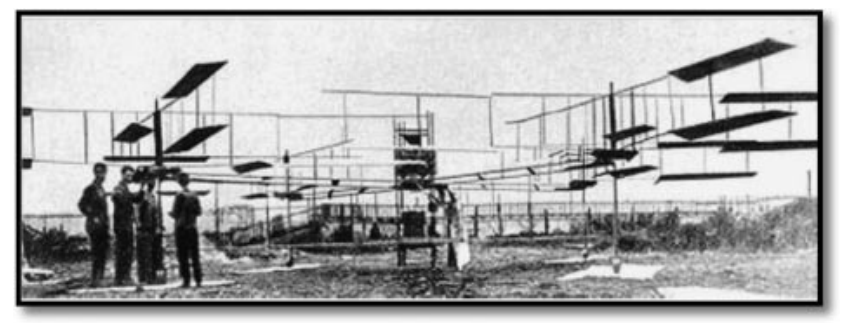

Fig. 1. The Breguet-Richet Quadrotor Gyroplane No. 1.2)

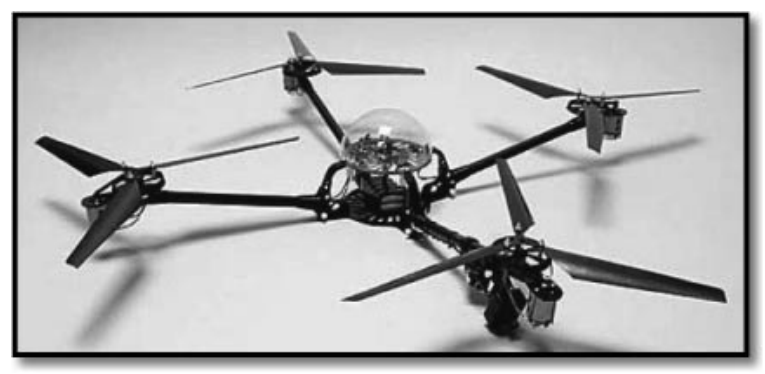

Fig. 2. Baseline Draganflyer X-Pro. ${ }^{3)}$

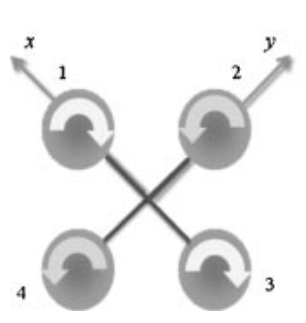

(a)

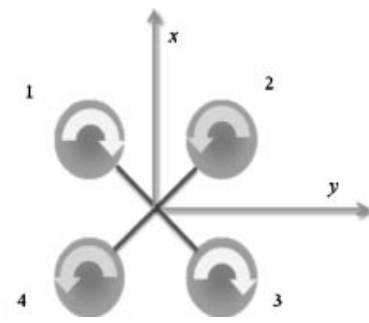

(b)
Fig. 3. a) Old coordinate system, b) New coordinate system.

with lateral motion and vice versa. In this research a new steering command using four motors together to accomplish roll and pitch commands is determined (Fig. 3b). This steering approach eliminates gyroscopic effects theoretically and then no interference appears between pitch and roll channels. This helps the vehicle to track the trajectory correctly. Arrows in below pictures indicate the direction of propellers rotation.

Table 1 shows the form of the paper.

With consideration of new coordinate system (Fig. 3b), new steering command is described as follows. The two pairs of propellers $(1,3)$ and $(2,4)$ turn in opposite directions. Increasing or decreasing the four propeller's speeds together generates vertical motion. If the counter-clockwise rotors increase their rotational speed, the resultant induced aerodynamic moment will cause the Quadrotor to turn clockwise (yawing motion). Slowing down the front rotors $(1,2)$ and at the same time speeding up the rear rotors $(3,4)$ produces negative pitch rotation and vice versa produces Positive pitch rotation. To produce Positive roll rotation must decrease the right rotors $(2,3)$ and at the same time increase the left rotors $(1,4)$ and vice versa produces negative roll rotation. In this manner the roll and pitch chan-
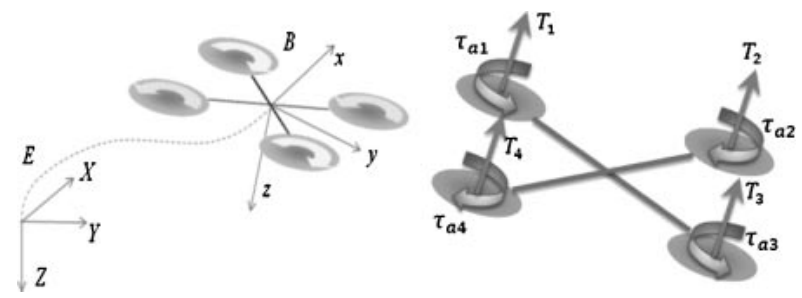

Fig. 4. a) Inertial coordinate system (E) and body coordinate system (B). b) thrust and aerodynamic moment acting on Quadrotor.

Table 1. Characteristic parameters of Quadrotor.

\begin{tabular}{cll}
\hline Parameter & Value & \multicolumn{1}{c}{ Unit } \\
\hline$m$ & 0.52 & $\mathrm{~kg}$ \\
$J_{r}$ & $6 \mathrm{e}-5$ & $\mathrm{~kg} \cdot \mathrm{m}^{2}$ \\
$b$ & $3.13 \mathrm{e}-5$ & $\mathrm{~N} \cdot \mathrm{s}^{2}$ \\
$d$ & $7.5 \mathrm{e}-7$ & $\mathrm{~N} \cdot \mathrm{m} \cdot \mathrm{s}^{2}$ \\
$I_{x x}$ & $6.23 \mathrm{e}-3$ & $\mathrm{~kg} \cdot \mathrm{m}^{2}$ \\
$I_{y y}$ & $6.23 \mathrm{e}-3$ & $\mathrm{~kg} \cdot \mathrm{m}^{2}$ \\
$I_{z z}$ & $1.12 \mathrm{e}-2$ & $\mathrm{~kg} \cdot \mathrm{m}^{2}$ \\
$l$ & 0.23 & $\mathrm{~m}$ \\
$\Omega_{n}$ & 201.8524 & $\mathrm{rad} / \mathrm{sec}$ \\
\hline
\end{tabular}

nel don't couple in together, because in pitch rotation, the gyroscopic effect of rotor 1 is neutralized by rotor 2 and gyroscopic effect of rotor 3 is neutralized by rotor 4 and then there will be no gyroscopic effects theoretically.

The Quadrotor is an under actuated mechanical system with $6 \mathrm{DOF}$ and only 4 actuators. The main forces and moments acting on it are those produced by the propellers (Fig. 4b). We can assume that the thrust $T_{i}$ and the aerodynamic moment $\tau_{a i}$ are proportional to the square of the propellers rotation speed. ${ }^{6}$ ) Arrows in (Fig. 4b) show the direction of aerodynamic moments. Thus we have:

$$
\begin{aligned}
& \tau_{a i}=d \cdot\left(\Omega_{i}\right)^{2} \\
& T_{i}=b \cdot\left(\Omega_{i}\right)^{2} \quad i=1,2,3,4
\end{aligned}
$$

$\Omega_{i}$ is the $i$ th motor rotational speed number. We express $\Omega$ as follows:

$$
\Omega=\Omega_{1}+\Omega_{3}-\Omega_{2}-\Omega_{4}
$$

In which:

$$
\left[\begin{array}{l}
\Omega_{1} \\
\Omega_{2} \\
\Omega_{3} \\
\Omega_{4}
\end{array}\right]=\left[\begin{array}{l}
\Omega_{n} \\
\Omega_{n} \\
\Omega_{n} \\
\Omega_{n}
\end{array}\right]+\left[\begin{array}{rrrr}
1 & 1 & -1 & -1 \\
1 & -1 & -1 & +1 \\
1 & -1 & +1 & -1 \\
1 & 1 & +1 & +1
\end{array}\right]\left[\begin{array}{c}
\Delta \Omega_{Z} \\
\Delta \Omega_{\phi} \\
\Delta \Omega_{\theta} \\
\Delta \Omega_{\psi}
\end{array}\right]
$$

Where $\Omega_{n}$ is the nominal rotational speed of each rotor and $\Delta \Omega_{Z}$ is variation in motors speed to accomplish climbing flight, $\Delta \Omega_{\phi}, \Delta \Omega_{\theta}, \Delta \Omega_{\psi}$ are variation in speed of motors provided by controller to accomplish roll, pitch and yaw motion respectively. Considering inertial coordinate system (E) and body coordinate system (B) as shown in (Fig. 4a) and using Euler angles parameterization, the airframe orientation in space is given by a rotation $T_{\psi \theta \phi}$ from $\mathrm{E}$ to $\mathrm{B}$, where this rotation is described as follow: 


$$
T_{\psi \theta \phi}=\left[\begin{array}{ccc}
c(\psi) c(\theta) & c(\theta) s(\psi) & -s(\theta) \\
c(\psi) s(\theta) s(\phi)-s(\psi) c(\phi) & s(\psi) s(\theta) s(\phi)+c(\psi) c(\phi) & c(\theta) s(\phi) \\
c(\psi) s(\theta) c(\phi)+s(\psi) s(\phi) & s(\psi) s(\theta) c(\phi)-c(\psi) s(\phi) & c(\theta) c(\phi)
\end{array}\right]
$$

where $c(\psi)=\cos (\psi)$ and $s(\psi)=\sin (\psi)$. The equation (5) relates the body angular velocity to rate of change of Euler angles.

$$
\left[\begin{array}{c}
\omega_{x} \\
\omega_{y} \\
\omega_{z}
\end{array}\right]=\left[\begin{array}{c}
\dot{\phi}-\sin (\theta) \dot{\psi} \\
\cos (\phi) \dot{\theta}+\sin (\phi) \cos (\theta) \dot{\psi} \\
-\sin (\phi) \dot{\theta}+\cos (\phi) \cos (\theta) \dot{\psi}
\end{array}\right]
$$

Dynamic model is derived using Newton-Euler formalism. From Newton's Second law ${ }^{7)}$ it can be deduced that

$$
\vec{F}=\frac{\mathrm{d}(m \vec{V})}{\mathrm{d} t} \quad \vec{M}=\frac{\mathrm{d}(\vec{H})}{\mathrm{d} t}
$$

where $F$ represents the summation of all externally applied forces, $M$ represents the sum of all applied torques, and $H$ is the angular momentum. For a rigid body, angular momentum may be defined as:

$$
\vec{H}=I . \vec{\omega}
$$

\subsection{The translational equation of motion}

External forces applied to Quadrotor are thrust of all motors $(T)$, body drag forces due to motion of body $(D)$ and gravitational force $(\mathrm{mg})$. In the inertial frame, the forces can be described as:

$$
\vec{F}_{\text {inertial }}=\left[\begin{array}{c}
0 \\
0 \\
m g
\end{array}\right]+T_{\psi, \theta, \phi}^{-1} \cdot\left[\begin{array}{c}
D_{x} \\
D_{y} \\
D_{z}-T
\end{array}\right]
$$

Therefore, the equations of motion in the inertial frame are represented as follows:

$$
\left[\begin{array}{l}
a_{X} \\
a_{Y} \\
a_{Z}
\end{array}\right]=\frac{1}{m} \cdot\left[\begin{array}{c}
c \theta \cdot c \psi \cdot D_{x}+(s \phi \cdot s \theta \cdot c \psi-c \phi \cdot s \psi) \cdot D_{y}+(c \phi \cdot s \theta \cdot c \psi+s \phi \cdot s \psi) \cdot\left(D_{z}-T\right) \\
c \theta \cdot s \psi \cdot D_{x}+(s \phi \cdot s \theta \cdot s \psi-c \phi \cdot c \psi) \cdot D_{y}+(c \phi \cdot s \theta \cdot s \psi-s \phi \cdot c \psi) \cdot\left(D_{z}-T\right) \\
m g-s \theta \cdot D_{x}+(s \phi \cdot c \theta) \cdot D_{y}+(c \phi \cdot c \theta) \cdot\left(D_{z}-T\right)
\end{array}\right]
$$

\subsection{The rotational equation of motion}

The torques acting on the vehicle's body along pitch or roll axis is due to the difference between the thrust generated by each propeller on the other axis and the yawing moment is produced by the drag force, see (Fig. 4b). The momentum vector in body coordinate system is expressed as follow:

$$
\vec{M}=\left[\begin{array}{c}
\tau_{x} \\
\tau_{y} \\
\tau_{z}
\end{array}\right]=\left[\begin{array}{c}
\left(\left(T_{1}+T_{4}\right)-\left(T_{2}+T_{3}\right)\right) \cdot l \\
\left(\left(T_{1}+T_{2}\right)-\left(T_{3}+T_{4}\right)\right) \cdot l \\
\left(-\tau_{1}-\tau_{3}+\tau_{2}+\tau_{4}\right)
\end{array}\right]
$$

where $l$ is the distance between the helicopter center of gravity and the rotation center of each rotor. Angular momentum of Quadrotor includes two parts. First part is related to rotation of body and the other is related to rotors, then it can be expressed as follow:

$$
\vec{H}=\vec{H}_{\text {body }}+\vec{H}_{\text {blade }}
$$

Where:

$$
\vec{H}_{\text {body }}=\left[\begin{array}{ccc}
I_{x} & 0 & 0 \\
0 & I_{y} & 0 \\
0 & 0 & I_{z}
\end{array}\right] \cdot\left[\begin{array}{c}
\omega_{x} \\
\omega_{y} \\
\omega_{z}
\end{array}\right] \quad \vec{H}_{\text {blade }}=\left[\begin{array}{c}
0 \\
0 \\
J_{r} \Omega
\end{array}\right]
$$

The rate change of relative to inertial reference system is given by:

$$
\left.\left.\frac{d \vec{H}}{d t}\right)_{\text {inertial }}=\frac{d \vec{H}}{d t}\right)_{\text {body }}+\vec{\omega} \times \vec{H}
$$

then:

$$
\left.\frac{d(\vec{H})}{d t}\right)_{\text {inertial }}=\left[\begin{array}{c}
I_{x} \dot{\omega}_{x}+\omega_{y} \omega_{z}\left(I_{z}-I_{y}\right)+J_{r} \Omega \omega_{y} \\
I_{y} \dot{\omega}_{y}+\omega_{x} \omega_{z}\left(I_{x}-I_{z}\right)-J_{r} \Omega \omega_{x} \\
I_{z} \dot{\omega}_{z}+\omega_{x} \omega_{y}\left(I_{y}-I_{x}\right)
\end{array}\right]
$$

From Newton's Second law and equations (9) and (11), following equations are obtained:

$$
\left[\begin{array}{c}
\tau_{x} \\
\tau_{y} \\
\tau_{z}
\end{array}\right]=\left[\begin{array}{c}
I_{x} \dot{\omega}_{x}+\omega_{y} \omega_{z}\left(I_{z}-I_{y}\right)+J_{r} \Omega \omega_{y} \\
I_{y} \dot{\omega}_{y}+\omega_{x} \omega_{z}\left(I_{x}-I_{z}\right)-J_{r} \Omega \omega_{x} \\
I_{z} \dot{\omega}_{z}+\omega_{x} \omega_{y}\left(I_{y}-I_{x}\right)
\end{array}\right]
$$

Finally, the rotational motion can be rewritten in the equation (13), directly usable (after verification of its parameters) in simulation and control:

$$
\left[\begin{array}{c}
\dot{\omega}_{x} \\
\dot{\omega}_{y} \\
\dot{\omega}_{z}
\end{array}\right]=\left[\begin{array}{c}
\frac{\tau_{x}}{I_{x}}-\omega_{y} \omega_{z} \frac{\left(I_{z}-I_{y}\right)}{I_{x}}-\frac{J_{r} \Omega \omega_{y}}{I_{x}} \\
\frac{\tau_{y}}{I_{y}}-\omega_{x} \omega_{z} \frac{\left(I_{x}-I_{z}\right)}{I_{y}}+\frac{J_{r} \Omega \omega_{x}}{I_{y}} \\
\frac{\tau_{z}}{I_{z}}-\omega_{x} \omega_{y} \frac{\left(I_{y}-I_{x}\right)}{I_{z}}
\end{array}\right]
$$

\section{Guidance, Navigation, and Control (GNC)}

A Quadrotor flight software (part of the avionics suite) has the difficult job of directing and controlling the vehicle from its initial position to its final location autonomously. 


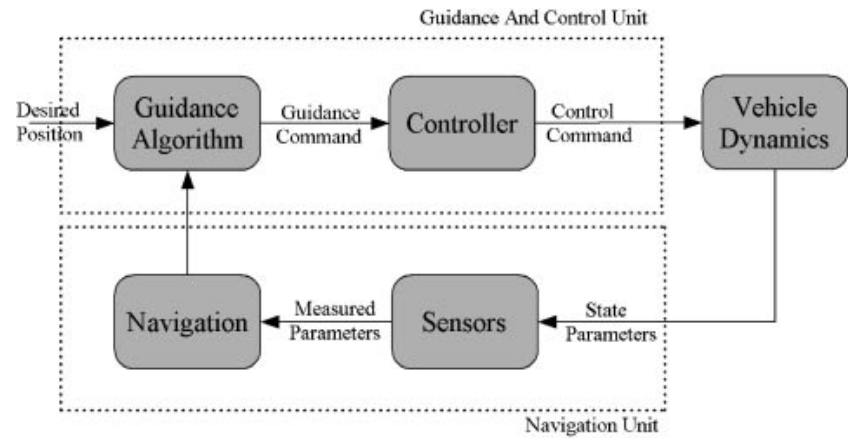

Fig. 5. Guidance, navigation, and control functions are performed by the flight computer (Flight software).

To accomplish this challenging task, the flight software must correctly perform three basic functions: guidance, navigation, and control (GNC) (Fig. 5).

Navigation is the process of determining the vehicle's position, velocity, and attitude in space. Guidance is the process of deciding how to steer to the desired target. Control is the process of implementing the guidance commands to determine appropriate rotational speed of motors. Guidance schemes are as varied as the systems they control. There is no generic guidance theory, the procedure of determining the flight trajectory and approach to the target is as follow:

1 - Doing the yaw motion and align the body $x$-axis with the LOS*.

2- Climbing and reach to desired height.

3- Doing the pitch motion and cruise to the target and hover in the specific height.

\subsection{Guidance}

The purpose of a guidance law is to provide automated trajectory following. The object state variables in the guidance system are vehicle velocity and position coordinates described by Newton translational equation of motion.

To accomplish that goal, the guidance law must have measurements of the current state of the vehicle, and be able to compare those measurements with the desired state of the vehicle. Based on those errors, the guidance law sends correction commands to a flight control system to re-acquire the desired trajectory automatically.

By having the coordinates of desired point, desired yaw motion for first phase of flight and desired height for second phase and desired longitudinal coordinate for third phase are computed with a series of mathematical relations. Then guidance block compare current measurements with the desired state of the vehicle and according to the phase of flight sends some commands to flight control system. If we have:

$$
\begin{aligned}
& \text { target coordinate }=\left(x_{\mathrm{des}}, y_{\mathrm{des}}, z_{\mathrm{des}}\right) \\
& \text { measured coordinate of Quadrotor }=\left(x_{\mathrm{m}}, y_{\mathrm{m}}, z_{\mathrm{m}}\right)
\end{aligned}
$$

Then the desired yaw angle is computed as follow:

$$
\psi_{\mathrm{des}}=\tan ^{-1}\left(y_{\mathrm{des}} / x_{\mathrm{des}}\right)
$$

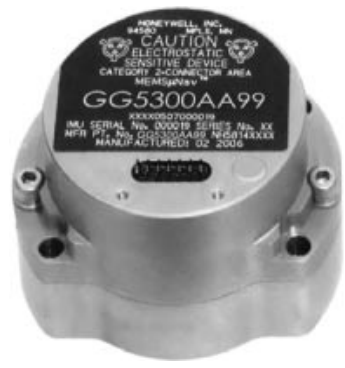

Fig. 6. Honeywell GG5300 MEMS Rate Gyro Package. ${ }^{9)}$

Guidance correction commands are computed as:

$$
\begin{aligned}
& \psi_{\mathrm{G}}=\psi_{\mathrm{des}}-\psi_{\mathrm{m}} \\
& h_{\mathrm{G}}=z_{\mathrm{des}}-z_{\mathrm{m}} \\
& x_{\mathrm{G}}=x_{\mathrm{des}}-x_{\mathrm{m}} \\
& y_{\mathrm{G}}=y_{\mathrm{des}}-y_{\mathrm{m}}
\end{aligned}
$$

\subsection{Navigation}

The flight control system must be developed to convert the correction commands into the physical control inputs used by the vehicle. The method is based on a pure inertial navigation system. Inertial navigation is a technique to compute estimates of the position and orientation of an object relative to a known starting pose using inertial measurements from accelerometers and gyroscopes. ${ }^{8}$ ) The IMU within the sensor unit contains a 3D rate gyroscope and a $3 \mathrm{D}$ linear accelerometer. The gyroscope and accelerometer are based on micro-machined electromechanical systems (MEMS) technology. Compared to traditional technology, MEMS devices are small, light, inexpensive, have low power consumption and short start-up times.

\section{Gyro model}

The MEMS gyroscope sensor used in the simulation, has one sensitive axis along which a physical quantity (specific angular velocity) is converted to an output voltage.

For this particular case a second order transfer function with cut-off frequency of $100 \mathrm{~Hz}$ was chosen (Honeywell GG5300 MEMS Rate Gyro Plant):

$$
T F_{\text {gyro }}=\frac{\omega_{\mathrm{r}}^{2}}{s^{2}+2 \zeta \omega_{\mathrm{r}} s+\omega_{\mathrm{r}}^{2}}
$$

Where:

$$
\begin{aligned}
& \omega_{\mathrm{r}}: \quad \text { response frequency }=100 \mathrm{~Hz} \\
& \zeta: \quad \text { damping }=\frac{1}{\sqrt{2}}
\end{aligned}
$$

\subsection{Control}

The basic purpose of the flight control system is to maintain the vehicle attitude commanded by the guidance program. This block senses the vehicle attitude via an inertial measurement system and commands the appropriate change in the rotation speed of rotors to achieve the commanded attitude while minimizing trajectory deviations. In order to design the Quadrotor controller, LQR method is chosen.

Linear quadratic regulator assumes that all the states are measurable and the system is observable and linear. Non- 
linear equations of motion have to be linearized to use the linear quadratic regulator controller.

$$
\dot{x}=A x(t)+B u(t)
$$

in which $u$ is the vector consist of control inputs (rotational speed of each motor) and $x$ is state variables vector. Considering the general equations for state-space system, cost function for a linearized system becomes:

$$
J=\frac{1}{2} \int_{t_{0}}^{t_{1}}\left[x^{\mathrm{T}}(t) Q x(t)+u^{\mathrm{T}}(t) R u(t)\right] \mathrm{d} t
$$

$Q$ is a positive-semi-defined state control matrix and it is important when defining which states are more important and which are less important. It means that, larger values of $Q$ generally results in the poles of the closed loop system being left in the s-plane so that the states decay faster to zero. $R$ is a positive-defined performance matrix also referred as the cost of inputs. In this case, the necessary condition for optimality of the time derivative of the Hamiltonian function is defined as:

$$
K=R^{-1} B^{\mathrm{T}} P
$$

Where $K$ is the optimal feedback gain and constant matrix $P$ is obtained from Algebraic Riccati equation:

$$
A^{\mathrm{T}} P+P A-P B R^{-1} B^{\mathrm{T}} P+Q=0
$$

Thus the solution of the quadratic problem reduces to solving Algebraic Riccati equation. The pair $(A, B)$ are completely controllable and the optimal control input is given as:

$$
u=-K x(t)
$$

If it is desired the final states of system converge to zero, it is

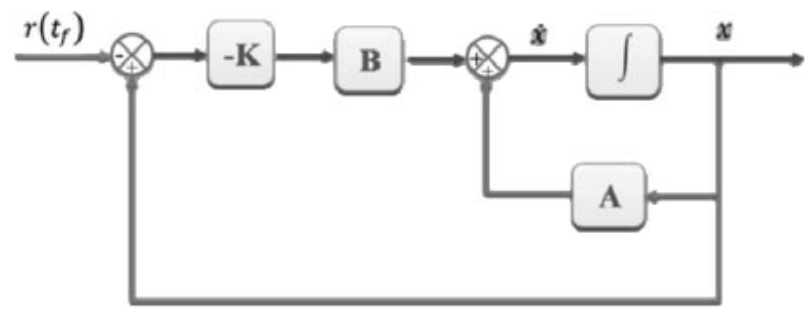

Fig. 7. The block diagram of optimal control law applied to linearized system.

sufficient to get feedback from the states of system via Eq. (20), otherwise if a non-zero number is desired, the final states $r\left(t_{\mathrm{f}}\right)$ must be subtracted from the current states of the system $x(t)$ and be multiplied by the matrix $k$ (Fig. 7), thus the optimal input to control the plant may be expressed as:

$$
u=-K\left[x(t)-r\left(t_{\mathrm{f}}\right)\right]
$$

As shown in Eq. (8), and Eq. (13), the model of the Quadrotor is non-linear. They have to be linearized to use the linear quadratic regulator controller by considering that the pair $(A, B)$ in Eq. (16), is completely controllable. For this purpose we design an optimal controller for first and second phase of flight and another for third phase of flight and another one in roll channel to damp disturbances in that channel. Simulation was performed on a linear model and then implemented on non-linear model.

1) Computation of optimal gain matrix in climbing flight:

To satisfy controllability condition, we consider $\psi$ and $Z$ and rate of them as state variables and $\Delta u_{Z}$ and $\Delta u_{\psi}$ for control inputs. Then $A, B$ matrixes are given as:

$$
\begin{aligned}
A & =\left[\begin{array}{cccc}
0 & 1 & 0 & 0 \\
0 & 0 & 0 & 0 \\
0 & 0 & 0 & \frac{1}{c \phi \cdot c \theta} \\
0 & 0 & 0 & 0
\end{array}\right]_{4 * 4} \\
B & =\left[\begin{array}{cc}
\frac{-(c \phi \cdot c \theta) \cdot\left(8 b \cdot\left(\Omega_{n}+\Delta \Omega_{Z}\right)\right)}{m} & \frac{-(c \phi \cdot c \theta) \cdot\left(8 b \cdot \Delta \Omega_{\psi}\right)}{m} \\
0 & 8 \cdot d \cdot\left(\Omega_{n}+\Delta \Omega_{Z}\right) / I_{z z}
\end{array}\right]
\end{aligned}
$$

Equilibrium point is considered at hover then the Euler angles and rate of them are zero and only $\Omega_{n}$ is acted to rotors. Therefore $A, B$ matrixes are obtained as bellow:

$$
A=\left[\begin{array}{llll}
0 & 1 & 0 & 0 \\
0 & 0 & 0 & 0 \\
0 & 0 & 0 & 1 \\
0 & 0 & 0 & 0
\end{array}\right]_{4 * 4} B=\left[\begin{array}{cc}
0 & 0 \\
-0.09720 & 0 \\
0 & 0 \\
0 & 0.1081
\end{array}\right]_{4 * 2}
$$

$$
Q=\left[\begin{array}{cccc}
100 & 0 & 0 & 0 \\
0 & 100 & 0 & 0 \\
0 & 0 & 100 & 0 \\
0 & 0 & 0 & 100
\end{array}\right]_{4 * 4} \quad R=\left[\begin{array}{ll}
1 & 0 \\
0 & 1
\end{array}\right]_{2 * 2}
$$

By solving Riccati equation, $P$ is computed and then replaced in Eq. (18), the optimal gain matrix is given by: 


$$
K=\left[\begin{array}{cccc}
-10.0000 & -17.4860 & 0 & 0 \\
0 & 0 & 10.0000 & 16.8824
\end{array}\right]
$$

2) Computation of optimal gain matrix in cruise flight:

To satisfy controllability condition, $X, Z$ and $\theta$ and rate of them are considered as the state variables and $\Delta u_{Z}$ and $\Delta u_{\theta}$ as the control inputs. Then $A, B$ matrixes are given as:

$$
\begin{aligned}
& A=\left[\begin{array}{cccccc}
0 & 1 & 0 & 0 & 0 & 0 \\
0 & 0 & 0 & 0 & {\left[-s \theta \cdot c \psi \cdot D_{x}+(s \phi \cdot c \theta \cdot c \psi) \cdot D_{y}+(c \phi \cdot c \theta \cdot c \psi) \cdot\left(D_{z}-T\right)\right] / m} & 0 \\
0 & 0 & 0 & 1 & 0 & 0 \\
0 & 0 & 0 & 0 & {\left[-c \theta \cdot D_{x}-(s \phi \cdot s \theta) \cdot D_{y}-(c \phi \cdot s \theta) \cdot\left(D_{z}-T\right)\right] / m} & 0 \\
0 & 0 & 0 & 0 & \left(s \phi \cdot s \theta \cdot \frac{\dot{\psi}}{c \phi}\right) & \frac{1}{c \phi} \\
0 & 0 & 0 & 0 & -c \theta \cdot \dot{\psi} \cdot \frac{\omega_{z} \cdot\left(I_{x x}-I_{z z}\right)+J_{r} \cdot \Omega}{I_{y y}}-c \phi \cdot s \theta \cdot \dot{\psi} \cdot \frac{\omega_{x} \cdot\left(I_{x x}-I_{z z}\right)}{I_{y y}} & 0
\end{array}\right] \\
& B=\left[\begin{array}{cc}
0 & 0 \\
-\frac{(c \phi \cdot s \theta \cdot c \psi+s \phi \cdot s \psi) \cdot\left(8 b \cdot\left(\Omega_{n}+\Delta \Omega_{Z}\right)\right)}{m} & -\frac{(c \phi \cdot s \theta \cdot c \psi+s \phi \cdot s \psi) \cdot\left(8 b \cdot \Delta \Omega_{\theta}\right)}{m} \\
0 & 0 \\
-\frac{(c \phi \cdot c \theta) \cdot\left(8 b \cdot\left(\Omega_{n}+\Delta \Omega_{Z}\right)\right)}{m} & -(c \phi \cdot c \theta) \cdot\left(8 b \cdot \Delta \Omega_{\theta}\right) / m \\
0 & 0 \\
-8 b \cdot l \cdot \Delta \Omega_{\theta} / I_{y y} & -8 \cdot b \cdot l \cdot\left(\Omega_{n}+\Delta \Omega_{Z}\right) / I_{y y}
\end{array}\right]
\end{aligned}
$$

Selecting hover flight condition as the operating point, Euler angles and their rates are zero, only $\Omega_{n}$ is acted to rotors, and the thrust vector is equal to weight of Quadrotor. Drag forces also are zero, therefore matrixes $A$ and $B$ are obtained as bellow:

$$
\begin{aligned}
A & =\left[\begin{array}{cccccc}
0 & 1 & 0 & 0 & 0 & 0 \\
0 & 0 & 0 & 0 & -9.81 & 0 \\
0 & 0 & 0 & 1 & 0 & 0 \\
0 & 0 & 0 & 0 & 0 & 0 \\
0 & 0 & 0 & 0 & 0 & 1 \\
0 & 0 & 0 & 0 & 0 & 0
\end{array}\right]_{6 * 6} \\
B & =\left[\begin{array}{cccccc}
0 & & 0 & \\
& 0 & & 0 \\
0 & & 0 & \\
-0.09720 & 0 & \\
0 & & 0 & \\
0 & & -1.8660
\end{array}\right]_{6 * 2} \\
Q & =\left[\begin{array}{cccccc}
0.01 & 0 & 0 & 0 & 0 & 0 \\
0 & 0.01 & 0 & 0 & 0 & 0 \\
0 & 0 & 10 & 0 & 0 & 0 \\
0 & 0 & 0 & 10 & 0 & 0 \\
0 & 0 & 0 & 0 & 0.01 & 0 \\
0 & 0 & 0 & 0 & 0 & 0.01
\end{array}\right]_{6 * 6}
\end{aligned}
$$

$$
R=\left[\begin{array}{cc}
0.1 & 0 \\
0 & 100
\end{array}\right]_{2 * 2}
$$

Therefore matrix $K$ for cruise and hovering flight is obtained as:

$K=$

$\left[\begin{array}{cccccc}0 & 0 & -10.00 & -17.486 & 0 & 0 \\ 0.010 & 0.0419 & 0 & 0 & -0.8156 & -0.9350\end{array}\right]$

3) Computation of optimal gain matrix for roll channel:

To satisfy controllability condition, roll angle $\varphi$ and its rate are considered as the state variables and $\Delta u_{\varphi}$ as control input. Then $A, B$ matrixes are obtained as:

$$
\begin{array}{rlrl}
A & =\left[\begin{array}{ll}
0 & 1 \\
0 & 0
\end{array}\right]_{2 * 2} & B & =\left[\begin{array}{c}
0 \\
8 b \cdot b \cdot l \cdot\left(\Omega_{n}+\Delta \Omega_{Z}\right) / I_{x x}
\end{array}\right]_{2 * 1} \\
A & =\left[\begin{array}{ll}
0 & 1 \\
0 & 0
\end{array}\right]_{2 * 2} & B & =\left[\begin{array}{c}
0 \\
1.8660
\end{array}\right]_{2 * 1} \\
Q & =\left[\begin{array}{cc}
1000 & 0 \\
0 & 1000
\end{array}\right]_{2 * 2} & R & =[1]_{1 * 1}
\end{array}
$$

matrix $K$ is obtained as:

$$
K=\left[\begin{array}{ll}
31.6228 & 32.1542
\end{array}\right]
$$

\section{Results}

The initial position of the Quadrotor is $(0,0,0)$. The simulation of the nonlinear model using LQR controller is 

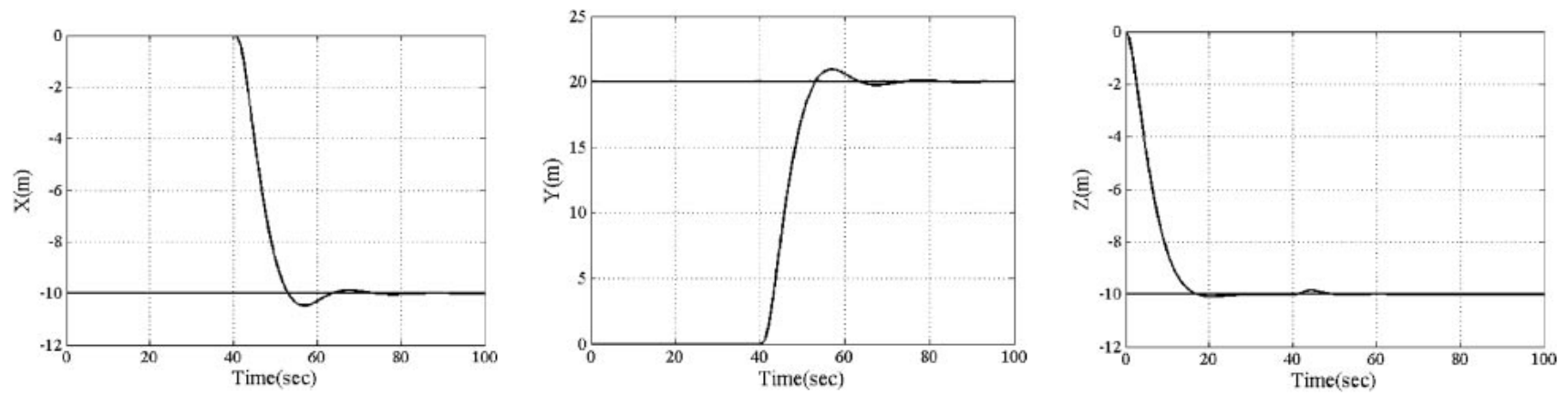

Fig. 8. Position of Quadrotor during performing of simulation.
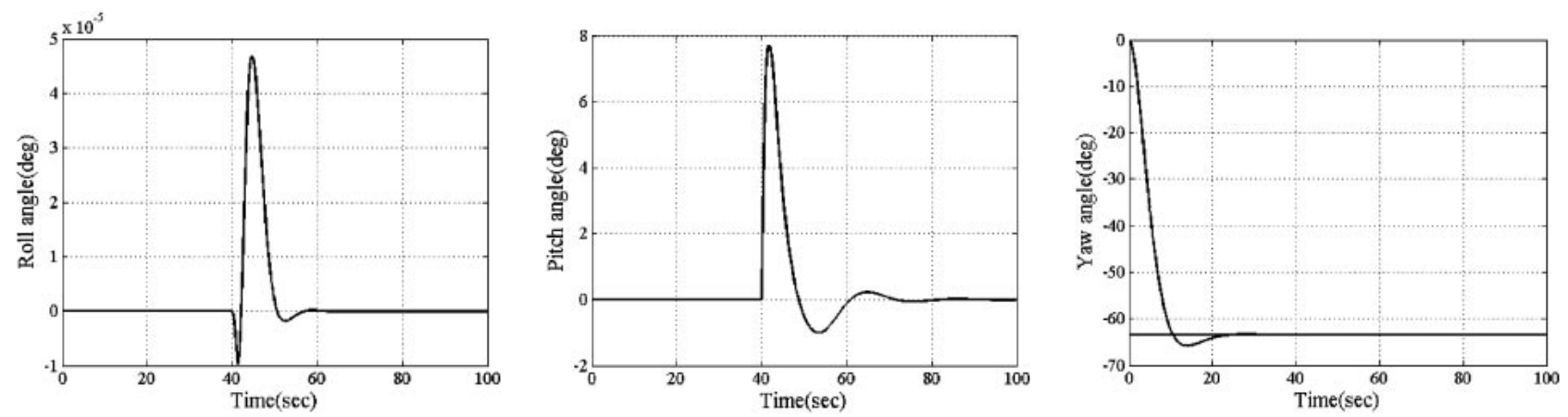

Fig. 9. Euler angles of Quadrotor during performing of simulation.

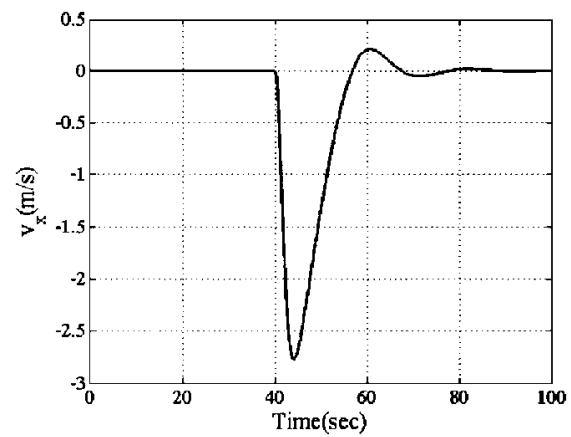

Fig. 10. Velocity in direction of motion.

addressed. The purpose is to reach to coordinate $(-10,20,-10)$. The results of simulation are shown in Figs. 8 and 9 imply that the Quadrotor has performed yaw motion and climbing in 20 seconds and approximately after 20 seconds the cruise flight is started and finally the Quadrotor reaches to final position and hovers on that height and does not have any motion. Control inputs are illustrated in Fig. 11.

Results indicate that responses are satisfactory and implemented controller properly executes the guidance commands. As shown in Fig. 10, the Quadrotor is able to fly from its initial position to its final location and hover on that height.

\section{Conclusion}

Quadrotor helicopters are popular as test beds for small UAV development. In this paper a guidance law using
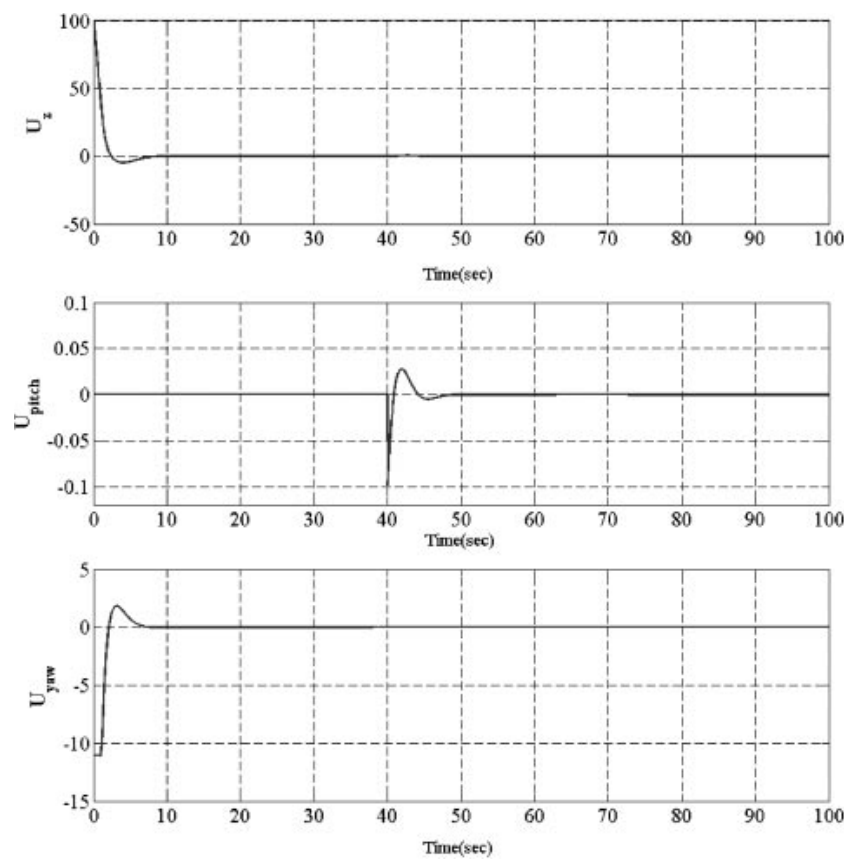

Fig. 11. Control inputs, $u_{z}, u_{\theta}, u_{\psi}$.

LQR control method is proposed to optimize the consumption of energy. The simulation results show that the proposed controller works effectively to minimize tracking error in addition to energy consumption. Our future work concerns the planning of trajectories that achieve specified objectives, and verify vehicle performance constraints. 


\section{References}

1) O'Brien, P. C.: Using a Robotic Helicopter to Fuel Interest in and Augment the Human Exploration of the Planet Mars, AIAA Space Conference 2003, Long Beach, California, pp. 1-11.

2) Leishman, J. G.: History of Helicopter Flight, http://www.glue.umd.edu/ leishman/Aero/history.html

3) http://www.draganfly.com/draganflyerxpro.php

4) Pounds, P., Mahony, R. and Corke, P.: Modeling and Control of a Quad-Rotor Robot, Proceedings of the Australasian Conference on Robotics and Automation, 2006.
5) Castillo, P., Dzul, A. and Lozano, R.: Real-Time Stabilization and Tracking of a Four-Rotor Mini Rotorcraft, IEEE Trans. Control Syst. Technol., 12 (2004), pp. 510-516.

6) Boubdallah, S., Murrieri, P. and Siegwart, R.: Design and Control of an Indoor Micro Quadrotor, ICRA 2004, New Orleans, USA, April 2004.

7) McLean, D.: Automatic Flight Control Systems, Prentice Hall, New York, 1990, Chap. 2.

8) Titterton, D. and Weston, J.: Strapdown Inertial Navigation Technology, 2nd ed., The Institution of Electrical Engineers, Steventage, UK, 2004.

9) www.honeywell.com 